\title{
Synthesis and antifungal activity of novel polyheterocyclic compounds containing fused 1,2,4-triazine moiety
}

\author{
Magdy A. Ibrahim,* Reda M. Abdel-Rahman, Ali M. Abdel-Halim, Salah S. Ibrahim, \\ and Hassan A. Allimony \\ Department of Chemistry, Faculty of Education, Ain Shams University, \\ Roxy, 11711, Cairo-Egypt \\ E-mail: magdy_ahmed1977@yahoo.com
}

\begin{abstract}
3-Amino-4-(4-chlorophenyl)-7-hydrazino-8H-pyrazolo[4,3-e][1,2,4]triazolo[1 ', ` -a]pyridine5-carbonitrile (4) was synthesized from 4-(4-chlorophenyl)-1,6-diamino-2-oxo-1,2-dihydropyridine-3,5-dicarbonitrile (1). Reaction of 4 with $\alpha, \beta$-bifunctional compounds gave pyrazolotriazinotriazolopyridines (8-14). The behavior of 4 towards condensation reactions with indole-2,3-dione in different media gave different products 15-16. Acetylation of 16 led to different products depending on the reaction conditions. Structures of the products have been deduced from analytical and spectral data (UV, IR, ${ }^{1} \mathrm{H} N M R,{ }^{13} \mathrm{C}$ NMR and mass spectra). Some of the products were screened for antifungal activity.
\end{abstract}

Keywords: Synthesis, o-diamine, triazolopyridine, pyrazolotriazinotriazolopyridine, fungicidal activity

\section{Introduction}

Polyfunctional pyridines are highly reactive reagents that have been used extensively in heterocyclic synthesis ${ }^{1-3}$ and that possess biological as well as pharmacological activity. ${ }^{4-6}$ Triazolopyridines are also interesting compounds due to their pronounced biological activity, as they can be used as antidepressants. ${ }^{7-8}$ Various 1,2,4-triazine derivatives are well known to possess an array of physiological activities, such as anticancer, muscle relaxant, hypnotic, anti-inflammatory, diuretic and antihypertensive activities. ${ }^{9-12}$

In continuation of our work in the area of fused 1,2,4-triazines ${ }^{13-19}$ and their heterocyclization via ring closing reactions with $\alpha, \beta$-bifunctional reagents, ${ }^{20-25}$ the present work aimed at the synthesis of fused heteropolycyclic nitrogen systems containing a fused 


\section{1,2,4-triazine moiety starting from 4-(4-chlorophenyl)-1,6-diamino-2-oxo-1,2- dihydropyridine-3,5-dicarbonitrile (1) and evaluation of their antifungal activity.}

\section{Results and Discussion}

The hydrazine derivative 4, as starting material for polyfused heterocyclic systems, was obtained from the reaction of 4-(4-chlorophenyl)-1,6-diaminopyridine-3,5-dicarbonitrile (1) with $\mathrm{CS}_{2} / \mathrm{KOH}$ to give thioxotriazolopyridinone 2 followed by hydrazinolysis. Also, methylation of 2 with $\mathrm{MeI} / \mathrm{KOH}$ produced the 2-methylthio derivative 3 which on hydrazinolysis afforded $4 .^{26-27}$ On the other hand, compound 4 was also obtained using alternative pathways. Treatment of diamine 1 with hydrazine hydrate afforded 4-(4chlorophenyl)-3,6,7-triamino-7H-pyrazolo [3,4-b]pyridine-5-carbonitrile (5). Heating an ethanolic solution of 5 with $\mathrm{CS}_{2} / \mathrm{KOH}$ at reflux yielded thioxopyrazolotriazolopyridine 6 which on methylation yielded the methylthio derivative 7. Hydrazinolysis of 6 and/or 7 furnished the hydrazino derivative 4 (Scheme 1). Compound 4 was confirmed by its elemental analysis and spectral data. The IR spectrum revealed the disappearance of the absorption band at $1669 \mathrm{~cm}^{-1}$ assigned to the $\mathrm{C}=\mathrm{O}$ group of the pyridinone ring in compound $\mathbf{1}$ and a new band was observed at $1637 \mathrm{~cm}^{-1}$ corresponding to an azomethine group in addition to the hydrazino group at 3469 and $3308 \mathrm{~cm}^{-1}$. Its ${ }^{13} \mathrm{C}$ NMR spectrum showed one signal at $\delta 117.1 \mathrm{ppm}$ corresponding to one cyano group. The mass spectrum of $\mathbf{4}$ showed a molecular ion peak at $m / e 340$ with the base peak at $m / e 285$, presumably due to the higher stability of the fused pyrazolopyridine system. 


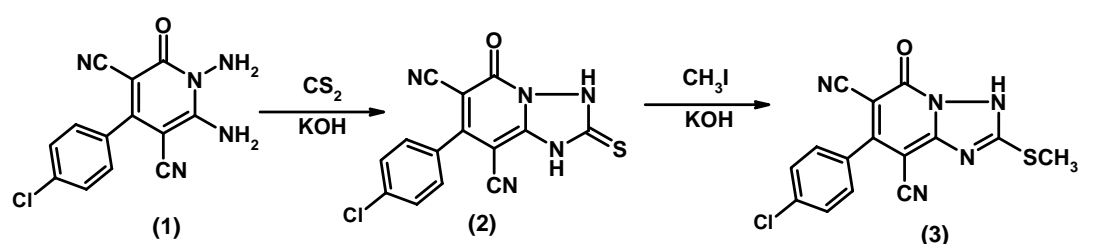

(1)

(2)

(3)

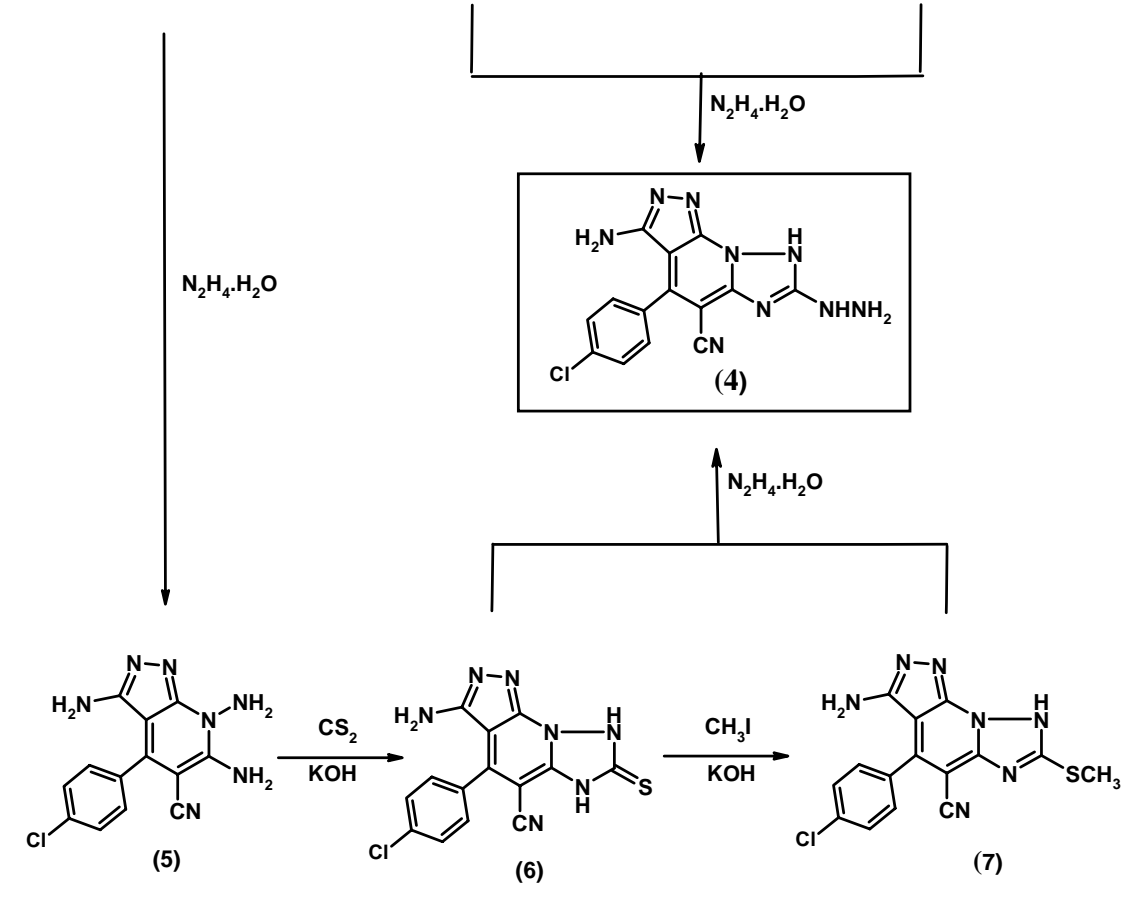

Scheme 1. Synthetic pathway for the preparation of target compound 4.

The target compound 4 was used for the synthesis of polyfused systems. Thus, fused triazinones 8a-c were obtained from cyclocondensation of $\mathbf{4}$ with $\alpha$-oxoacids, namely; glyoxalic, pyruvic, $p$-chlorostyrylglyoxalic acid in glacial acetic acid ${ }^{[28]}$ (Scheme 2).

The isomeric fused triazinones $\mathbf{9}$ and $\mathbf{1 0}$ were obtained from cyclocondensation of compound $\mathbf{4}$ with monochloroacetic acid and/or chloroacetyl chloride, ${ }^{29}$ respectively (Scheme 2). ${ }^{13} \mathrm{C}$ NMR spectra of compounds $\mathbf{9}$ and $\mathbf{1 0}$ showed characteristic signals at $\delta 35.7$ and 35.9 ppm assigned to $\mathrm{CH}_{2}$ carbons, respectively (Figure 1).

Perhydro fused 1,2,4-triazinotriazoles $\mathbf{1 1}$ and $\mathbf{1 2}$ were obtained from boiling compound $\mathbf{4}$ with phenacyl bromide and 1,2-dibromoethane ${ }^{30-31}$ in ethanolic $\mathrm{NaOH}(5 \%)$, respectively (Scheme 2). 
<smiles>N#Cc1c(-c2ccc(Cl)cc2)c2c(N)nnc-2n2c1nc1n2CCNN1</smiles>

(12)
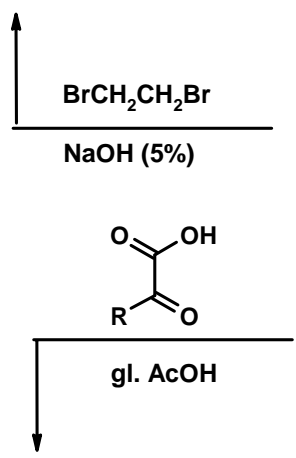<smiles></smiles>

(8 a-c)<smiles>N#Cc1c(-c2ccc(Cl)cc2)c2c(N)nnc-2n2c1nc1n2CC(c2ccccc2)=NN1</smiles>

(11)

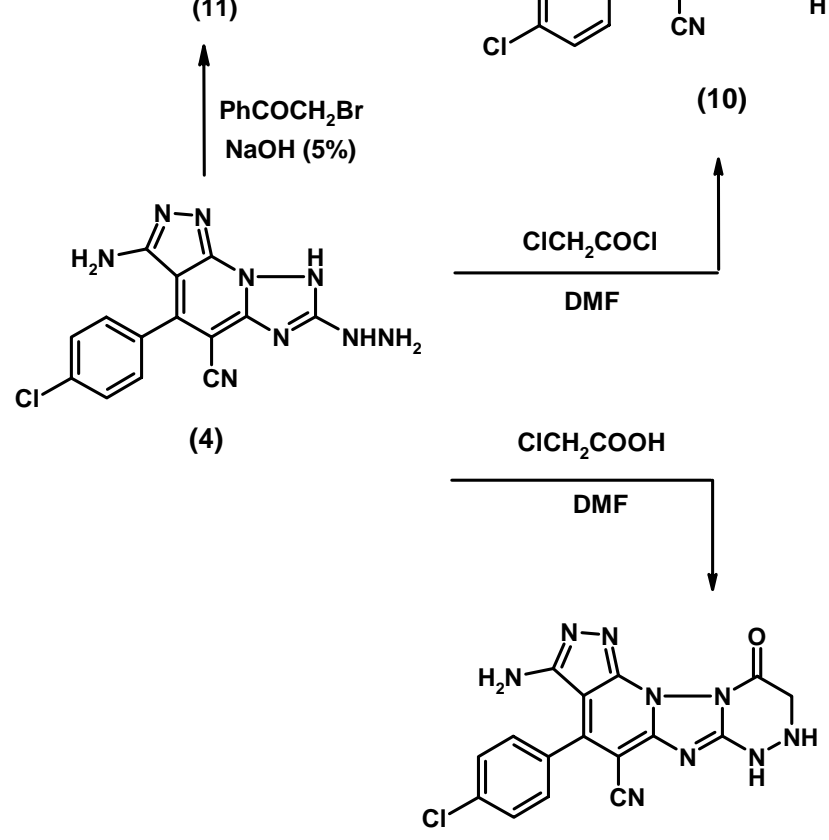

(9)

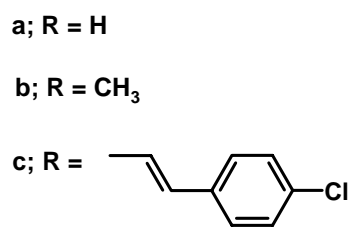

Scheme 2. Synthetic pathway for the preparation of compounds 8-12.

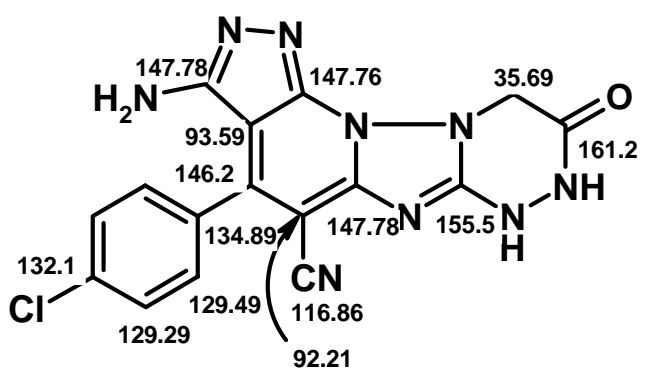

9

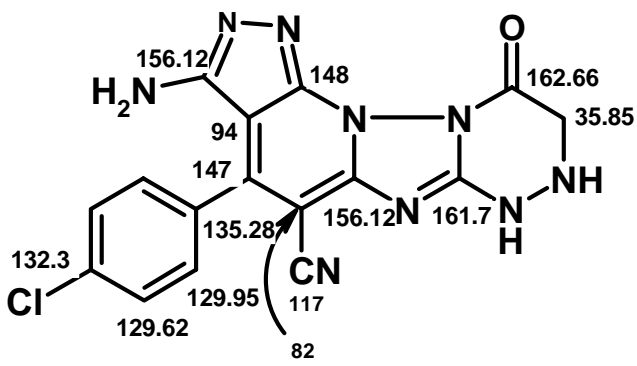

10

Figure 1. ${ }^{13} \mathrm{C}$ NMR of compounds 9 and $\mathbf{1 0}$. 
Reactions of 4 with $\alpha, \beta$-dicarbonyl compounds have been investigated. Thus, treatment of 4 with diethyl oxalate ${ }^{32}$ in boiling DMF produced the 9,10-dioxo derivative 13. Also, cyclocondensation of 4 with benzoin in glacial acetic acid yielded the 9,10-diphenyl derivative 14 (Scheme 3). On the other hand, cyclocondensation of compound 4 with indole2,3-dione in boiling DMF afforded 3-amino-4-(4-chlorophenyl)-7H-indolo[2,3-

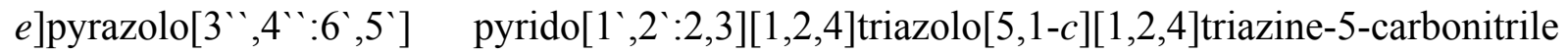
(15), while treatment of an equimolar ratio of 4 and indole-2,3-dione in ethanolic $\mathrm{NaOH}$ solution afforded 3-amino-9-(2-aminophenyl)-4-(4-chlorophenyl)-10-oxo-12-hydro-7H-

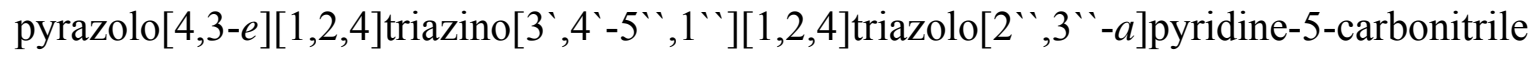

(16) (Scheme 3). Compound 15 was obtained authentically by cyclization of 16 in glacial acetic acid and a few drops of concentrated $\mathrm{H}_{2} \mathrm{SO}_{4}$, where the absorption band of $\mathrm{C}=\mathrm{O}$ group disappeared and showed co-identical IR spectra. Acetylation of compound $\mathbf{1 6}$ using acetic anhydride furnished the diacetyl derivative 17. The IR spectrum revealed the presence of new absorption bands at $1720,1655 \mathrm{~cm}^{-1}$ for two $\mathrm{C}=\mathrm{O}$ groups and at $3422 \mathrm{~cm}^{-1}$ for $\mathrm{NH}$ group (Scheme 3).

Finally, the behavior of compound $\mathbf{1 6}$ towards acetylation reactions has been studied under different conditions. Thus, refluxing 16 with glacial acetic acid afforded the monoacetyl derivative 18. However, when the reaction was carried out in boiling glacial acetic acid containing a few drops of acetic anhydride, the diacetyl derivative 19 was isolated (Scheme 4). Structures of mono- and diacetyl derivatives 18 and 19 were established form their elemental analysis and spectral data. IR spectrum of 18 showed an absorption band at 1764 $\mathrm{cm}^{-1}$ assigned to one $\mathrm{C}=\mathrm{O}$, while 19 showed two absorption bands at 1740 and $1702 \mathrm{~cm}^{-1}$ assigned to two $\mathrm{C}=\mathrm{O}$ functions. ${ }^{1} \mathrm{H}$ NMR of 18 showed a signal at $\delta 1.91 \mathrm{ppm}$, characteristic for one $\mathrm{COCH}_{3}$ group, while that of 19 showed two signals at $\delta 1.56$ and $1.92 \mathrm{ppm}$, characteristic for two $\mathrm{COCH}_{3}$ groups.

\section{Fungicidal activity}

Some new synthesized compounds were screened for their antifungal activities against two fungi, Alternaria alternata and Aspergillus niger using the disc diffusion method. ${ }^{33-34}$ The tested compounds were dissolved in DMF [which has no inhibition activity] to get $1 \mathrm{mg} / \mathrm{ml}$ solution. The antibiotic flucanazole was used as standard antifungal reference. The inhibition zones of the microbial growth surrounding the filter paper disc $(2.5 \mathrm{~mm})$ were measured in millimeters at the end of an incubation period at $30{ }^{\circ} \mathrm{C}$ for 3 days (Table 1 ).

All the tested compounds showed variable activities toward the two species in comparison to the standard flucanazole which revealed that these compounds are biologically active due to the presence of different heterocycles and functional groups.

From the results obtained, it is clear that most of the tested compounds showed moderate activity toward the tested fungi except compound $\mathbf{1 3}$ showed higher activity towards Alternaria alternata fungi which mainly due to the expected dihydroxy structure (Table 1) 

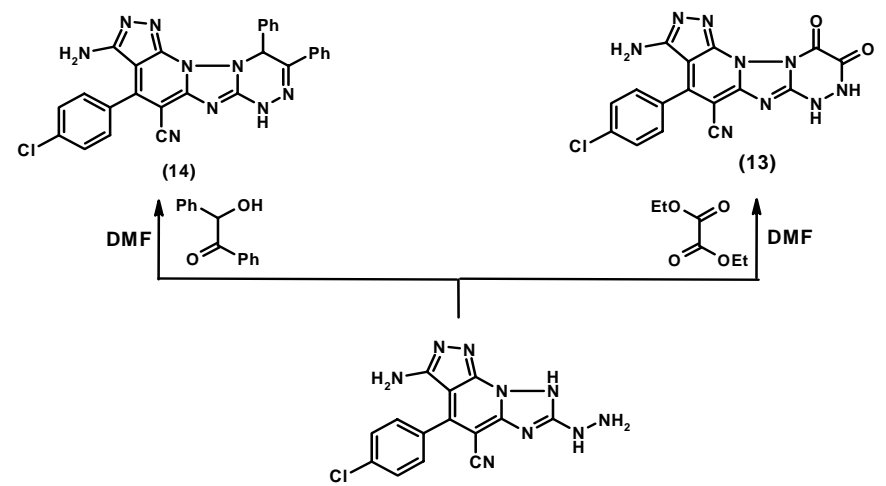

(4)
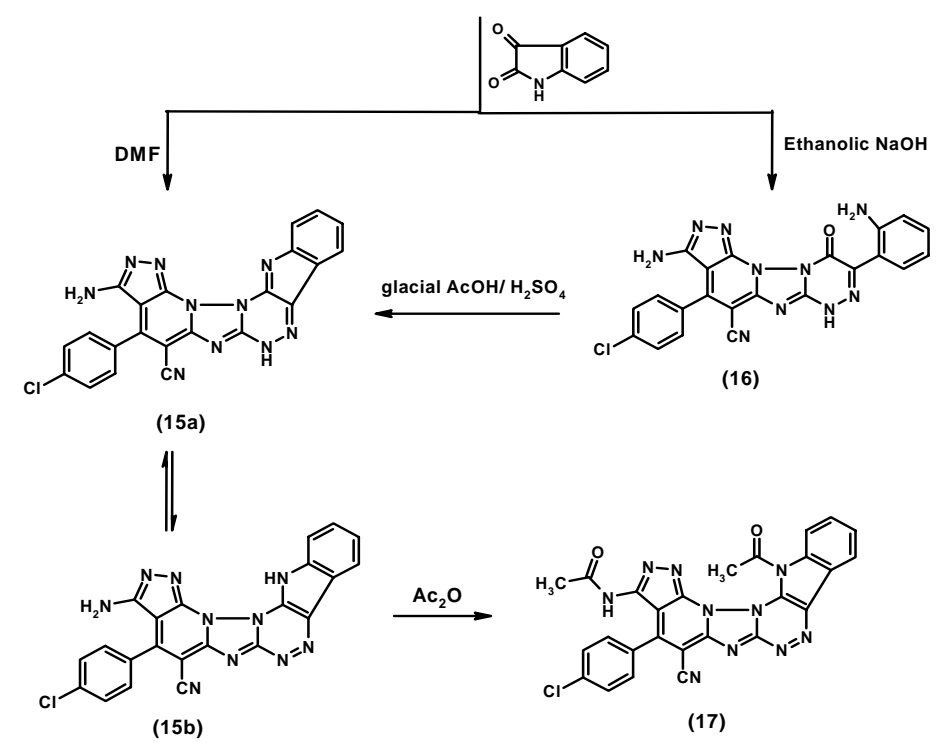

Scheme 3. Synthetic pathway for the preparation of compounds 13-17. 
<smiles>N#Cc1c(-c2ccc(Cl)cc2)c2c(N)nnc-2n2c1nc1[nH]nc(-c3ccccc3N)c(=O)n12</smiles>

(16)

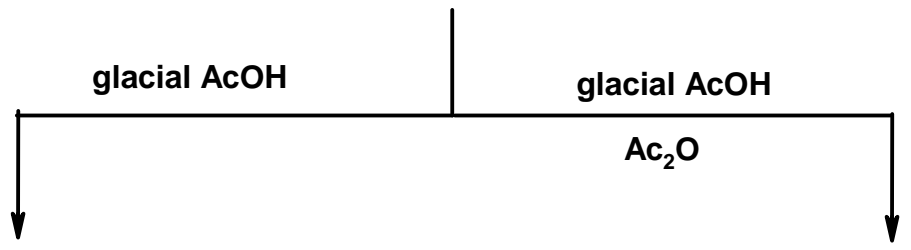<smiles></smiles><smiles></smiles>

Scheme 4. Synthetic pathway for the preparation of compounds 18-19.

Table 1. Fundicidal activity of some of the prepared compounds 2-19.

\begin{tabular}{ccc}
\hline \multirow{2}{*}{ Compd. No. } & \multicolumn{2}{c}{ Diameter of inhibition zone $(\mathrm{mm})$} \\
\cline { 2 - 3 } & Alternaria alternata & Aspergillus niger \\
\hline $\mathbf{4}$ & ++ & + \\
$\mathbf{5}$ & + & + \\
$\mathbf{6}$ & ++ & + \\
$\mathbf{8 c}$ & ++ & ++ \\
$\mathbf{9}$ & + & ++ \\
$\mathbf{1 0}$ & + & ++ \\
$\mathbf{1 3}$ & +++ & + \\
$\mathbf{1 5}$ & ++ & ++ \\
$\mathbf{1 6}$ & ++ & + \\
$\mathbf{1 9}$ & ++ & ++ \\
(Flucanazole) & +++ & +++ \\
\hline
\end{tabular}

Lower active $=+($ inhibition zone $1-10 \mathrm{~mm})$, Moderately active $=++($ inhibition zone 11 -25 $\mathrm{mm})$ and High active $=+++($ inhibition zone $>25 \mathrm{~mm})$. 


\section{Experimental Section}

General Procedures. Melting points are uncorrected and were recorded in open capillary tubes on a Stuart SMP3 melting point apparatus. Infrared spectra were recorded on FT-IR Bruker Vector 22 spectrophotometer using $\mathrm{KBr}$ wafer technique. UV absorption spectra (DMF) were recorded on a Jasco model (V-550) UV spectrophotometer. ${ }^{1} \mathrm{H}$ NMR spectra were measured on Gemini spectrometer $200 \mathrm{MHz}$ and $\mathrm{AC}$ spectrometer $250 \mathrm{MHz}$ using DMSO- $d_{6}$ as solvent and TMS (chemical shift in $\delta \mathrm{ppm}$ ) as an internal standard. ${ }^{13} \mathrm{C}$ NMR spectra were measured on AC spectrometer $250 \mathrm{MHz}$ using DMSO as solvent and TMS (chemical shift in $\delta \mathrm{ppm}$ ) as an internal standard. Mass spectra were obtained using gas chromatography GCMS qp 1000 ex Schimadzu instrument mass spectrometer $(70 \mathrm{eV})$. Elemental microanalyses were performed at the Cairo University Microanalytical Center. 4(4-chlorophenyl)-1,6-diamino-2-oxo-1,2-dihydropyridine-3,5-dicarbonitrile (1) has been prepared according to the reported method. ${ }^{35}$

\section{7-(4-Chlorophenyl)-5-oxo-2-thioxo-1,2,3,5-tetrahydro[1,2,4]triazolo[1,5-a]pyridine-6,8-} di-carbonitrile (2). A mixture of $1(2.85 \mathrm{~g}, 0.01 \mathrm{~mol})$ and carbon disulfide $(0.60 \mathrm{~mL}, 0.01$ $\mathrm{mol})$ in ethanolic potassium hydroxide $(10 \%, 100 \mathrm{~mL})$ was refluxed for 4 hours, after cooling the reaction mixture was poured onto ice-HCl. The solid obtained was filtered, washed several times with water and crystallized from methanol to give 2 as yellow crystals, yield $2.13 \mathrm{~g}$ (65\%), mp $>300{ }^{\circ} \mathrm{C}$. UV $\lambda_{\max }(\log \varepsilon): 346(4.18), 275 \mathrm{~nm}(2.405) . \mathrm{IR}\left(\mathrm{KBr}, \mathrm{cm}^{-1}\right): 3212,3184$ $(2 \mathrm{NH}), 2216(2 \mathrm{C} \equiv \mathrm{N}), 1674(\mathrm{C}=\mathrm{O}), 1284(\mathrm{C}=\mathrm{S}) .{ }^{1} \mathrm{H}$ NMR $\left(\delta, \mathrm{DMSO}_{6}\right): 7.64(\mathrm{~d}, 2 \mathrm{H}, \mathrm{Ar}-\mathrm{H}$, $\mathrm{J}=8.4 \mathrm{~Hz}), 7.82\left(\mathrm{~d}, 2 \mathrm{H}\right.$, Ar-H, J = 8.4 Hz), $9.24\left(\mathrm{~s}, 1 \mathrm{H}, \mathrm{NH}\right.$ exchangeable with $\left.\mathrm{D}_{2} \mathrm{O}\right), 10.36$ ppm (s, 1H, NH exchangeable with $\mathrm{D}_{2} \mathrm{O}$ ). Anal. Calcd. for $\mathrm{C}_{14} \mathrm{H}_{6} \mathrm{ClN}_{5} \mathrm{OS}$ (327.76): C, 51.26; H, 1.83; N, 21.36; S, 9.76. Found C, 51.12; H, 1.80; N, 20.95; S, 9.55.

7-(4-Chlorophenyl)-2-methylthio-5-oxo-,3,5-dihydro[1,2,4]triazolo[1,5-a]pyridine-6,8-dicarbonitrile (3). A mixture of 2 (1.64 g, $0.005 \mathrm{~mol})$ and methyl iodide $(0.31 \mathrm{~mL}, 0.005 \mathrm{~mol})$ in ethanolic potassium hydroxide $(10 \%, 100 \mathrm{~mL})$ was refluxed for 4 hours, after cooling the reaction mixture was poured onto ice- $\mathrm{HCl}$. The solid obtained was filtered, wash several times with water and crystallized from aqueous DMF to give 3 as yellow crystals, yield 1.14 $\mathrm{g}(67 \%), \mathrm{mp}>300^{\circ} \mathrm{C}$. IR $\left(\mathrm{KBr}, \mathrm{cm}^{-1}\right): 3209(\mathrm{NH}), 2960\left(\mathrm{CH}_{3}\right), 2220(2 \mathrm{C} \equiv \mathrm{N}), 1672(\mathrm{C}=\mathrm{O})$, $1640(\mathrm{C}=\mathrm{N}) .{ }^{1} \mathrm{H}$ NMR $\left(\delta, \mathrm{DMSO}_{6}\right): 4.14\left(\mathrm{~s}, 3 \mathrm{H}, \mathrm{CH}_{3}\right), 8.31(\mathrm{~d}, 2 \mathrm{H}, \mathrm{Ar}-\mathrm{H}), 8.42$ (d, 2H, Ar$\mathrm{H}), 8.61 \mathrm{ppm}\left(\mathrm{s}, 1 \mathrm{H}, \mathrm{NH}\right.$ exchangeable with $\left.\mathrm{D}_{2} \mathrm{O}\right)$.

4-(4-Chlorophenyl)-3,6,7-triamino-7H-pyrazolo[3,4-b]pyridine-5-carbonitrile (5). A mixture of 1 ( $2.85 \mathrm{~g}, 0.01 \mathrm{~mol})$ and hydrazine hydrate $(5 \mathrm{~mL})$ was refluxed for 6 hours, after cooling the reaction mixture was poured onto ice. The solid obtained was filtered and crystallized from aqueous DMF to give 5 as yellow crystals, yield $1.44 \mathrm{~g}(48 \%), \mathrm{mp}>300{ }^{\circ} \mathrm{C}$. UV $\lambda_{\max }(\log \varepsilon) ; 395$ (3.00), 350 (4.5), $275 \mathrm{~nm}(4.3)$. IR $\left(\mathrm{KBr}, \mathrm{cm}^{-1}\right): 3470,3303,3188$ (3 $\left.\mathrm{NH}_{2}\right), 2214(\mathrm{C} \equiv \mathrm{N}), 1637(\mathrm{C}=\mathrm{N}) .{ }^{1} \mathrm{H} \mathrm{NMR}\left(\delta, \mathrm{DMSO}-d_{6}\right): 4.59\left(\mathrm{bs}, 4 \mathrm{H}, 2 \mathrm{NH}_{2}\right.$ exchangeable 
with $\left.\mathrm{D}_{2} \mathrm{O}\right), 5.29$ (bs, $1 \mathrm{H}, \mathrm{NH}$ exchangeable with $\left.\mathrm{D}_{2} \mathrm{O}\right), 5.76$ (bs, $1 \mathrm{H}, \mathrm{NH}$ exchangeable with $\left.\mathrm{D}_{2} \mathrm{O}\right), 7.56$ (d, 2H, Ar-H, J = 8.6 Hz), 7.67 ppm (d, 2H, Ar-H, J = 8.6 Hz). MS (Int.\%): 299 (100), 300 (48.05), 284 (22.77), 189 (7.11), 172 (5.49), 111 (23.09). Anal. Calcd. for $\mathrm{C}_{13} \mathrm{H}_{10} \mathrm{ClN}_{7}$ (299.72): C, 52.05; H, 3.34; N, 32.69. Found C, 51.85; H, 3.42; N, 32.35.

3-Amino-4-(4-chlorophenyl)-7-thioxo-7,8-dihydro-6H-pyrazolo[4,3-e][1,2,4]triazolo[1,5a] pyridine-5-carbonitrile (6). A mixture of 5 (2.99 g, $0.01 \mathrm{~mol})$ and carbon disulfide $(0.60$ $\mathrm{mL}, 0.01 \mathrm{~mol})$ in ethanolic potassium hydroxide $(10 \%, 100 \mathrm{~mL})$ was refluxed for 4 hours, after cooling the reaction mixture was poured onto ice-HCl. The solid obtained was filtered, washed several times with water and crystallized from aqueous DMF to give 6 as yellow crystals, yield $2.49 \mathrm{~g}(73 \%), \mathrm{mp}>300^{\circ} \mathrm{C}$. UV $\lambda_{\max }(\log \varepsilon) ; 410$ (3.1), 355 (4.7), $278 \mathrm{~nm}(4.4)$. IR $\left(\mathrm{KBr}, \mathrm{cm}^{-1}\right)$ : 3469, 3305, $3187\left(2 \mathrm{NH}, \mathrm{NH}_{2}\right), 2214(\mathrm{C} \equiv \mathrm{N}) 1633(\mathrm{C}=\mathrm{N}), 1290(\mathrm{C}=\mathrm{S})$. Anal. Calcd. for $\mathrm{C}_{14} \mathrm{H}_{8} \mathrm{ClN}_{5} \mathrm{~S}$ (341.78): C, 49.20; H, 2.36; N, 28.69; S, 9.38. Found C, 48.97; H, 2.24; N, 28.75; S, 9.26.

3-Amino-4-(4-chlorophenyl)-7-methylthio-7,8-dihydro-6H-pyrazolo[4,3-e][1,2,4]triazolo [1,5-a]pyridine-5-carbonitrile (7). A mixture of 6 (1.71 g, $0.005 \mathrm{~mol})$ and methyl iodide $(0.31 \mathrm{~mL}, 0.005 \mathrm{~mol})$ ethanolic potassium hydroxide $(10 \%, 50 \mathrm{~mL})$ was refluxed for 6 hours, after cooling the reaction mixture was poured onto ice-HCl. The solid obtained was filtered, washed several times with water and crystallized from aqueous DMF to give 7 as yellow crystals, yield $1.22 \mathrm{~g}(69 \%), \mathrm{mp}>300{ }^{\circ} \mathrm{C}$. IR $\left(\mathrm{KBr}, \mathrm{cm}^{-1}\right): 3469,3317,3219\left(\mathrm{NH}_{2}, \mathrm{NH}\right)$, $2925\left(\mathrm{CH}_{3}\right), 2216(\mathrm{C} \equiv \mathrm{N}), 1623(\mathrm{C}=\mathrm{N})$ and 1459, $1422\left(\right.$ def. $\left.\mathrm{CH}_{3}\right) \mathrm{cm}^{-1} .{ }^{1} \mathrm{H}$ NMR $(\delta$, DMSO$\left.d_{6}\right): 3.96\left(\mathrm{~s}, 3 \mathrm{H}, \mathrm{CH}_{3}\right), 5.62\left(\mathrm{bs}, 2 \mathrm{H}, \mathrm{NH}_{2}\right.$ exchangeable with $\left.\mathrm{D}_{2} \mathrm{O}\right), 8.21(\mathrm{~d}, 2 \mathrm{H}, \mathrm{Ar}-\mathrm{H}, \mathrm{J}=8.2$ $\mathrm{Hz}), 8.42(\mathrm{~d}, 2 \mathrm{H}, \mathrm{Ar}-\mathrm{H}, \mathrm{J}=8.2 \mathrm{~Hz}), 8.95 \mathrm{ppm}\left(\mathrm{s}, 1 \mathrm{H}, \mathrm{NH}\right.$ exchangeable with $\left.\mathrm{D}_{2} \mathrm{O}\right)$.

\section{Hydrazinolysis of 2, 3, 6 and/or 7. Formation of 4}

A mixture of 2, 3, 6 and/or $7(0.005 \mathrm{~mol})$ and hydrazine hydrate $(5 \mathrm{~mL})$ refluxed for 16 hours, after cooling the reaction mixture was poured onto ice-AcOH. The solid obtained was filtered and crystallized from DMF to give 4 as yellow crystals, mp $>300{ }^{\circ} \mathrm{C}$. UV $\lambda_{\max }(\log \varepsilon)$ : 347(4.22), $271 \mathrm{~nm}$ (2.49). IR ( $\left.\mathrm{KBr}, \mathrm{cm}^{-1}\right): 3469,3308,3200\left(2 \mathrm{NH}_{2}, 2 \mathrm{NH}\right), 2209(\mathrm{C} \equiv \mathrm{N}), 1628$ $(\mathrm{C}=\mathrm{N}) \mathrm{cm}^{-1} .{ }^{1} \mathrm{H}$ NMR $\left(\delta\right.$, DMSO-d $\left.{ }_{6}\right): 5.21\left(\mathrm{~s}, 6 \mathrm{H}, 2 \mathrm{NH}\right.$ and $\left.2 \mathrm{NH}_{2}\right), 7.53(\mathrm{~d}, 2 \mathrm{H}, \mathrm{Ar}-\mathrm{H}), 7.64$ $\operatorname{ppm}(\mathrm{d}, 2 \mathrm{H}, \mathrm{Ar}-\mathrm{H}) .{ }^{13} \mathrm{C} \mathrm{NMR}\left(\delta, \mathrm{DMSO}_{6}\right): 92.76\left(\mathrm{C}_{5}-\mathrm{CN}\right), 93.47\left(\mathrm{C}_{3 \mathrm{a}}\right), 117.12(\mathrm{C} \equiv \mathrm{N})$, 129.11, 129.57, 132.58, 134.79 (6C of aryl carbons), $146.69\left(\mathrm{C}_{4}\right), 148.64\left(\mathrm{C}_{3}\right), 154.79\left(\mathrm{C}_{5 \mathrm{a}}\right)$ and 162.34 ppm $\left(\mathrm{C}_{7}\right.$ and $\left.\mathrm{C}_{9 \mathrm{a}}\right)$. MS (Int.\%): 340 (0.63), 313 (1.27), 285 (100), 228 (1.88), 113 (5.34) and 56 (3.16). Anal. Calcd. for $\mathrm{C}_{14} \mathrm{H}_{10} \mathrm{ClN}_{9}$ (339.75): C, 49.49; H, 2.96; N, 37.10. Found C, 48.65; H, 2.62; N, 37.35.

3-Amino-4-(4-chlorophenyl)-9-(un)substituted-10-oxo-12hydro-7H-pyrazolo[4,3-e][1,2,4] triazino[3 3 , ${ }^{-5}$ “,1“] $][1,2,4]$ triazolo[2“,3“-a]pyridine-5-carbonitriles (8a-c). A mixture of $4(1.70 \mathrm{~g}, 0.005 \mathrm{~mol})$ and $\alpha$-oxoacids such as glyoxalic, pyruvic and $p$-chlorostyrylglyoxalic acids $(0.005 \mathrm{~mol})$ in glacial acetic acid $(40 \mathrm{~mL})$ was refluxed for 4 hours, after cooling the reaction mixture was poured onto ice. The solid obtained was filtered and crystallized to give 
8a-c. Compound 8a crystallized from DMF as yellow crystals, yield $1.02 \mathrm{~g} \mathrm{(54 \% ),} \mathrm{mp} \mathrm{>} 300$ ${ }^{\circ} \mathrm{C}$. IR $\left(\mathrm{KBr}, \mathrm{cm}^{-1}\right): 3382,3199\left(\mathrm{NH}_{2}, \mathrm{NH}\right), 2224(\mathrm{C} \equiv \mathrm{N}), 1700(\mathrm{C}=\mathrm{O}), 1624(\mathrm{C}=\mathrm{N}) . \mathrm{MS}$ (Int.\%): 284 (3.85), 267 (1.13), 173 (1.35), 149 (7.3), 73 (100), 69 (20.95), 55 (13.62); Anal. Calcd. for $\mathrm{C}_{16} \mathrm{H}_{8} \mathrm{ClN}_{9} \mathrm{O}$ (377.75): C, 50.87; H, 2.13; N, 33.37. Found C, 50.61; H, 2.03; N, 33.08. Compound 8b crystallized from DMF as yellow crystals, yield $1.1 \mathrm{~g}(56 \%), \mathrm{mp}>300$ ${ }^{\circ} \mathrm{C}$. IR ( $\left.\mathrm{KBr}\right) \mathrm{cm}^{-1}:$ 3462, 3315, $3195\left(\mathrm{NH}_{2}, \mathrm{NH}\right), 2215(\mathrm{C} \equiv \mathrm{N}), 1702(\mathrm{C}=\mathrm{O}), 1625(\mathrm{C}=\mathrm{N}) \mathrm{cm}^{-}$ 1. ${ }^{1} \mathrm{H}$ NMR $\left(\delta\right.$, DMSO-d $\left.d_{6}\right): 2.7\left(\mathrm{~s}, 3 \mathrm{H}, \mathrm{CH}_{3}\right), 6.09$ (s, 2H, $\mathrm{NH}_{2}$ exchangeable with $\left.\mathrm{D}_{2} \mathrm{O}\right), 8.32$ $(\mathrm{d}, 2 \mathrm{H}, \mathrm{Ar}-\mathrm{H}), 8.43(\mathrm{~d}, 2 \mathrm{H}, \mathrm{Ar}-\mathrm{H}), 12.71 \mathrm{ppm}\left(\mathrm{bs}, 1 \mathrm{H}, \mathrm{NH} \rightleftharpoons \mathrm{OH}\right.$ exchangeable with $\left.\mathrm{D}_{2} \mathrm{O}\right)$. MS (Int.\%): 395 (0.26), 335 (8.68), 285 (100), 270 (4.85) and 193 (4.33) Anal. Calcd. for $\mathrm{C}_{17} \mathrm{H}_{10} \mathrm{ClN}_{9} \mathrm{O}$ (391.78): C, 52.12; H, 2.57; N, 32.18. Found C, 51.88; H, 2.40; N, 32.11.; Compound 8c crystallized from DMF as yellow crystals, yield $1.68 \mathrm{~g}(62 \%), \mathrm{mp}>300{ }^{\circ} \mathrm{C}$. UV $\lambda_{\max }(\log \varepsilon): 349$ (2.95), $278 \mathrm{~nm}$ (1.92). IR (KBr, cm $\left.{ }^{-1}\right): 3467,3314,3144\left(\mathrm{NH}_{2}, \mathrm{NH}\right)$, $2217(\mathrm{C} \equiv \mathrm{N}), 1701(\mathrm{C}=\mathrm{O}), 1623(\mathrm{C}=\mathrm{N})$. Anal. Calcd. for $\mathrm{C}_{24} \mathrm{H}_{13} \mathrm{Cl}_{2} \mathrm{~N}_{9} \mathrm{O}$ (514.34): $\mathrm{C}, 56.09 ; \mathrm{H}$, 2.55; N, 24.49. Found C, 56.61; H, 2.83; N, 25.01.

\section{3-Amino-4-(4-chlorophenyl)-10-oxo-12-hydro-7H,8H,9H-pyrazolo[4,3-e][1,2,4]triazino}

$[3,4-5 “, 1 “][1,2,4]$ triazolo[2“,3“-a]pyridine-5-carbonitrile (9). A mixture of 4 (1.70 g, $0.005 \mathrm{~mol})$ and monochloroacetic acid $(0.47 \mathrm{~g}, 0.005 \mathrm{~mol})$ in DMF $(40 \mathrm{~mL})$ was refluxed for 4 hours, after cooling the reaction mixture was poured onto ice. The solid obtained was filtered and crystallized from DMF to give 9 as yellow crystals, yield $1.44 \mathrm{~g} \mathrm{(76 \% ),} \mathrm{mp}>300$ ${ }^{\circ} \mathrm{C}$. IR $\left(\mathrm{KBr}, \mathrm{cm}^{-1}\right): 3329,3303\left(\mathrm{NH}_{2}, \mathrm{NH}\right), 2926\left(\mathrm{CH}_{2}\right), 2212(\mathrm{C} \equiv \mathrm{N}), 1639(\mathrm{C}=\mathrm{O}), 1555$ $(\mathrm{C}=\mathrm{N})$.

${ }^{1} \mathrm{H}$ NMR ( $\delta$, DMSO-d 6 ): 2.89 (s, 2H, $\left.\mathrm{CH}_{2}\right), 5.19$ (s, 2H, NH$), 7.4$ (d, 2H, 2Ar-H), 7.6 (d, 2H, Ar-H), 11.85 ppm (bs, 2H, 2NH). ${ }^{13} \mathrm{C}$ NMR ( $\delta$, DMSO-d 6$): 35.69\left(\mathrm{CH}_{2}\right), 92.21\left(\mathrm{C}_{5}-\mathrm{CN}\right)$, $93.59\left(\mathrm{C}_{3 \mathrm{a}}\right), 116.86(\mathrm{C} \equiv \mathrm{N}), 129.19,129.29,129.49,132.11$ and 134.89 (6C of aryl carbons), 146.22 $\left(\mathrm{C}_{4}\right), 147.76\left(\mathrm{C}_{12 \mathrm{a}}\right), 147.78\left(\mathrm{C}_{3}\right.$ and $\left.\mathrm{C}_{5 \mathrm{a}}\right), 155.58\left(\mathrm{C}_{6 \mathrm{a}}\right)$ and $161.21 \mathrm{ppm}\left(\mathrm{C}_{10}\right.$ as $\left.\mathrm{C}=\mathrm{O}\right)$. Anal. Calcd. for $\mathrm{C}_{16} \mathrm{H}_{10} \mathrm{ClN}_{9} \mathrm{O}$ (379.77): C, 50.60; H, 2.65; N, 33.19. Found $\mathrm{C}, 50.27 ; \mathrm{H}$, $2.43 ; \mathrm{N}, 32.82$.

\section{3-Amino-4-(4-chlorophenyl)-9-oxo-12-hydro-7H,8H,10H-pyrazolo[4,3-e][1,2,4]triazino} $\left[3,4{ }^{`}-5 “, 1 `\right][1,2,4]$ triazolo[2“,3“-a]pyridine-5-carbonitrile (10). A mixture of 4 (1.70 g, $0.005 \mathrm{~mol})$ and chloroacetyl chloride $(0.40 \mathrm{~mL}, 0.005 \mathrm{~mol})$ in DMF $(40 \mathrm{~mL})$ was refluxed for 4 hours, after cooling the reaction mixture was poured onto ice. The solid obtained was filtered and crystallized from DMF to give $\mathbf{1 0}$ as yellow crystals, yield $1.23 \mathrm{~g}(65 \%), \mathrm{mp}>$ $300{ }^{\circ} \mathrm{C}$. IR (KBr, cm $\left.{ }^{-1}\right): 3466,3310,3210\left(\mathrm{NH}_{2}, 2 \mathrm{NH}\right), 2945,2832\left(\mathrm{CH}_{2}\right), 2209(\mathrm{C} \equiv \mathrm{N}), 1700$ $(\mathrm{C}=\mathrm{O}), 1632(\mathrm{C}=\mathrm{N}), 1493,1421\left(\right.$ def. $\left.\mathrm{CH}_{2}\right) .{ }^{1} \mathrm{H}$ NMR $(\delta$, DMSO-d $): 2.75\left(\mathrm{~s}, 2 \mathrm{H}, \mathrm{CH}_{2}\right), 5.3$ $\left(\mathrm{s}, 2 \mathrm{H}, \mathrm{NH}_{2}\right), 7.5(\mathrm{~d}, 2 \mathrm{H}, 2 \mathrm{Ar}-\mathrm{H}), 7.9(\mathrm{~d}, 2 \mathrm{H}, 2 \mathrm{Ar}-\mathrm{H})$ and $11.90 \mathrm{ppm}(\mathrm{bs}, 2 \mathrm{H}, 2 \mathrm{NH}) .{ }^{13} \mathrm{C} \mathrm{NMR}$ $\delta$ : $35.85\left(\mathrm{CH}_{2}\right), 82\left(\mathrm{C}_{5}-\mathrm{CN}\right), 94\left(\mathrm{C}_{3 \mathrm{a}}\right), 117(\mathrm{C} \equiv \mathrm{N}), 129.62,129.95,132.39$ and $135.28(6 \mathrm{C}$ of aryl carbons), $147\left(\mathrm{C}_{4}\right), 148\left(\mathrm{C}_{12 \mathrm{a}}\right), 156.12\left(\mathrm{C}_{3}\right.$ and $\left.\mathrm{C}_{5 \mathrm{a}}\right), 161.7\left(\mathrm{C}_{6 \mathrm{a}}\right)$ and $162.66 \mathrm{ppm}\left(\mathrm{C}_{10}\right.$ as $\mathrm{C}=\mathrm{O}$ ). Anal. Calcd. for $\mathrm{C}_{16} \mathrm{H}_{10} \mathrm{ClN}_{9} \mathrm{O}$ (379.77): C, 50.60; H, 2.65; N, 33.19. Found C, 50.42; H, 2.31; N, 32.94. 


\section{3-Amino-4-(4-chlorophenyl)-9-phenyl-12-hydro-7H,10H-pyrazolo[4,3-e][1,2,4]triazino} $\left[3^{`}, 4-5 `, 1 ”\right][1,2,4]$ triazolo [2“,3“-a] pyridine-5-carbonitrile (11). A mixture of 4 (1.70 g, $0.005 \mathrm{~mol})$ and phenacyl bromide $(1 \mathrm{~g}, 0.005 \mathrm{~mol})$ in ethanolic $\mathrm{NaOH}(5 \%, 100 \mathrm{~mL})$ was refluxed for 4 hours, after cooling the reaction mixture was acidified with conc. $\mathrm{HCl}$. The solid obtained was filtered and crystallized from DMF to give $\mathbf{1 1}$ as yellow crystals, yield $1.12 \mathrm{~g}(51 \%), \mathrm{mp}>300{ }^{\circ} \mathrm{C}$. IR $\left(\mathrm{KBr}, \mathrm{cm}^{-1}\right): 3470,3315,3140\left(\mathrm{NH}_{2}, \mathrm{NH}\right), 2942,2831\left(\mathrm{CH}_{2}\right)$, $2214(\mathrm{C} \equiv \mathrm{N}), 1625(\mathrm{C}=\mathrm{N})$. Anal. Calcd. for $\mathrm{C}_{22} \mathrm{H}_{14} \mathrm{ClN}_{9}$ (439.87): C, 60.07; H, 3.21; N, 28.66. Found C, 59.75; H, 3.13; N, 28.28.

\section{3-Amino-4-(4-chlorophenyl)-12-hydro-7H,8H,9H,10H-pyrazolo[4,3-}

e][1,2,4] triazino[3`4`-5“,1“] $[1,2,4]$ triazolo[2“,3“-a]pyridine-5-carbonitrile (12). A mixture of $4(1.70 \mathrm{~g}, 0.005 \mathrm{~mol})$ and 1,2-dibromoethane $(0.43 \mathrm{~mL}, 0.005 \mathrm{~mol})$ in ethanolic $\mathrm{NaOH}(5 \%, 50 \mathrm{~mL})$ was refluxed for 4 hours, after cooling the reaction mixture was acidified with conc. $\mathrm{HCl}$. The solid obtained was filtered and crystallized from DMF to give $\mathbf{1 2}$ as yellow crystals, yield $1.04 \mathrm{~g}(57 \%), \mathrm{mp}>300^{\circ} \mathrm{C}$. UV $\lambda_{\max }(\log \varepsilon): 350(4.50), 275(4.25) \mathrm{nm}$. IR $\left(\mathrm{KBr}, \mathrm{cm}^{-1}\right)$ : 3468, 3313, $3207\left(\mathrm{NH}_{2}, 2 \mathrm{NH}\right), 2924,2840\left(\mathrm{CH}_{2}\right), 2215(\mathrm{C} \equiv \mathrm{N}), 1625(\mathrm{C}=\mathrm{N})$. ${ }^{1} \mathrm{H}$ NMR $\left(\delta\right.$, DMSO-d $\left.d_{6}\right): 3.3\left(\mathrm{~s}, 4 \mathrm{H}, 2 \mathrm{CH}_{2}\right), 5.93\left(\mathrm{bs}, 4 \mathrm{H}, \mathrm{NH}_{2}\right.$ and $2 \mathrm{NH}$ exchangeable by $\left.\mathrm{D}_{2} \mathrm{O}\right), 8.28(\mathrm{~d}, 2 \mathrm{H}, \mathrm{Ar}-\mathrm{H})$ and $8.53(\mathrm{~d}, 2 \mathrm{H}, \mathrm{Ar}-\mathrm{H})$. Anal. Calcd. for $\mathrm{C}_{16} \mathrm{H}_{12} \mathrm{ClN}_{9}$ (365.79): C, 52.49; H, 3.28; N, 34.45. Found C, 52.61; H, 3.43; N, 34.15.

\section{3-Amino-4-(4-chlorophenyl)-9,10-dioxo-12hydro-7H,8H-pyrazolo[4,3-e][1,2,4]triazino} $\left[3,4{ }^{`}-5 “, 1 `\right][1,2,4]$ triazolo[2“,3“-a]pyridine-5-carbonitrile (13). A mixture of 4 (1.70 g, $0.005 \mathrm{~mol})$ and diethyl oxalate $(0.68 \mathrm{~mL}, 0.005 \mathrm{~mol})$ in DMF $(40 \mathrm{~mL})$ was refluxed for 8 hours, after cooling the reaction mixture was poured onto ice. The solid obtained was filtered and crystallized from DMF to give 13 as yellow crystals, yield $1.2 \mathrm{~g}(61 \%), \mathrm{mp}>300^{\circ} \mathrm{C}$. $(\mathrm{KBr}$,

$\left.\mathrm{cm}^{-1}\right): 3477,3321,3147\left(\mathrm{NH}_{2}, 2 \mathrm{NH}\right), 2217(\mathrm{C} \equiv \mathrm{N}), 1711(\mathrm{C}=\mathrm{O}), 1629(\mathrm{C}=\mathrm{N}) .{ }^{1} \mathrm{H}$ NMR $(\delta$, DMSO- $\left.d_{6}\right): 5.24\left(\mathrm{~s}, 2 \mathrm{H}, \mathrm{NH}_{2}\right), 7.52-7.73(\mathrm{~m}, 4 \mathrm{H}, \mathrm{Ar}-\mathrm{H})$ and $11.88(\mathrm{~s}, 2 \mathrm{H}, \mathrm{NH}$ and $\mathrm{OH}$ of 1,2,4-triazine). ${ }^{13} \mathrm{C}$ NMR $\left(\delta\right.$, DMSO- $\left.d_{6}\right): 92.15\left(\mathrm{C}_{5}-\mathrm{CN}\right), 93.52\left(\mathrm{C}_{3 \mathrm{a}}\right), 116.90(\mathrm{C} \equiv \mathrm{N}) ; 129.19$, $129.49,132.05,134.87$ (6C of aryl carbons), $146.15\left(\mathrm{C}_{4}\right), 146.18\left(\mathrm{C}_{12 \mathrm{a}}\right), 146.22\left(\mathrm{C}_{5 \mathrm{a}}\right), 147.75$ $\left(\mathrm{C}_{3}\right), 155.62\left(\mathrm{C}_{9}\right.$ and $\left.\mathrm{C}_{6 \mathrm{a}}\right), 161.19\left(\mathrm{C}_{10}\right.$ as $\left.\mathrm{C}=\mathrm{O}\right)$.

\section{3-Amino-4-(4-chlorophenyl)-9,10-diphenyl-12-hydro-7H,10H-pyrazolo[4,3-e][1,2,4]} triazino[3`,4-5“,1“][1,2,4]triazolo[2“,3“-a]pyridine-5-carbonitrile (14). A mixture of 4 $(1.70 \mathrm{~g}, 0.005 \mathrm{~mol})$ and benzoin $(1.06 \mathrm{~g}, 0.005 \mathrm{~mol})$ in DMF $(40 \mathrm{~mL})$ was refluxed for 8 hours, after cooling the reaction mixture was poured onto ice. The solid obtained was filtered and crystallized from DMF to give 14 as yellow crystals, yield $1.68 \mathrm{~g}(65 \%), \mathrm{mp}>300{ }^{\circ} \mathrm{C}$. IR $\left(\mathrm{KBr}, \mathrm{cm}^{-1}\right)$ : 3470, 3315, $3142\left(\mathrm{NH}_{2}, \mathrm{NH}\right), 2922,2850\left(\mathrm{CH}_{2}\right) 2215(\mathrm{C} \equiv \mathrm{N}), 1626(\mathrm{C}=\mathrm{N})$. ${ }^{1} \mathrm{H}$ NMR $\left(\delta\right.$, DMSO-d $\left.d_{6}\right): 5.5$ (s, 2H, NH$\left.{ }_{2}\right), 7.6-7.9$ (m, 15H, Ar-H and $\mathrm{CH}$ of 1,2,4-triazin-5$\mathrm{yl})$ and $11.9 \mathrm{ppm}(\mathrm{s}, 1 \mathrm{H}, \mathrm{NH}) .{ }^{13} \mathrm{C} \mathrm{NMR}\left(\delta, \mathrm{DMSO}-d_{6}\right): 92\left(\mathrm{C}_{5}-\mathrm{CN}\right), 94\left(\mathrm{C}_{3 \mathrm{a}}\right), 117.98(\mathrm{C} \equiv \mathrm{N}$ and $\left.\mathrm{C}_{10}\right), 130.14-136.41$ (18C of aryl carbons), $147\left(\mathrm{C}_{4}\right.$ and $\left.\mathrm{C}_{12 \mathrm{a}}\right), 148\left(\mathrm{C}_{3}\right), 156\left(\mathrm{C}_{5 \mathrm{a}}\right), 162$ ( $\left.\mathrm{C}_{6 \mathrm{a}}\right), 195.69$ (C9). MS (Int.\%): 461 (2.99), 285 (100), 270 (7.83), 178 (2.35), 174 (6.17), 160 
(4.61). Anal. Calcd. for $\mathrm{C}_{28} \mathrm{H}_{18} \mathrm{ClN}_{9}$ (515.97): C, 65.12; H, 3.49; N, 24.42. Found C, 64.61; H, 3.43; N, 24.35.

3-Amino-4-(4-chlorophenyl)-15-hydro-7H-pyrazolo[4,3-e ]indolo[2,3-5`,6 $][1,2,4]$ triazino $\left[3{ }^{`}, 4{ }^{`} 5^{\prime}, 1 `\right][1,2,4]$ triazolo[2`,3“-a]pyridine-5-carbonitrile (15). A mixture of 4 (1.70 g, $0.005 \mathrm{~mol})$ and isatine $(0.74 \mathrm{~g}, 0.005 \mathrm{~mol})$ in DMF $(40 \mathrm{~mL})$ was refluxed for 8 hours, the reaction mixture was cooled and poured onto ice. The solid obtained was filtered and crystallized from DMF to give 15 as yellow crystals, yield $1.06 \mathrm{~g}(47 \%), \mathrm{mp}>300{ }^{\circ} \mathrm{C}$. IR $\left(\mathrm{KBr}, \mathrm{cm}^{-1}\right): 3468,3316,3179\left(\mathrm{NH}_{2}, \mathrm{NH}\right), 2218(\mathrm{C} \equiv \mathrm{N}), 1624(\mathrm{C}=\mathrm{N}) .{ }^{1} \mathrm{H}$ NMR $(\delta$, DMSO$\left.d_{6}\right): 5.3\left(\mathrm{~s}, 2 \mathrm{H}, \mathrm{NH}_{2}\right), 7.5-7.9(\mathrm{~m}, 8 \mathrm{H}, \mathrm{Ar}-\mathrm{H})$ and $11.9 \mathrm{ppm}(\mathrm{s}, 1 \mathrm{H}, \mathrm{NH}) .{ }^{13} \mathrm{C} \mathrm{NMR} \delta: 92\left(\mathrm{C}_{5^{-}}\right.$ $\mathrm{CN}), 93\left(\mathrm{C}_{3 \mathrm{a}}\right), 117(\mathrm{C} \equiv \mathrm{N}), 129.36-135.29$ (10C of aryl carbons), $146\left(\mathrm{C}_{4}\right), 147\left(\mathrm{C}_{15 \mathrm{a}}\right), 152$ $\left(\mathrm{C}_{3}\right), 156\left(\mathrm{C}_{5 \mathrm{a}}\right), 157\left(\mathrm{C}_{6 \mathrm{a}}\right), 161.67\left(\mathrm{C}_{13 \mathrm{a}}\right), 163 \mathrm{ppm}\left(\mathrm{C}_{8 \mathrm{a}}\right)$. Anal. Calcd. for $\mathrm{C}_{22} \mathrm{H}_{11} \mathrm{ClN}_{10}$ (515.97): C, 58.61; H, 2.46; N, 31.07. Found C, 58.82; H, 2.30; N, 30.74.

3-Amino-9-(2-aminophenyl)-4-(4-chlorophenyl)-10-oxo-12-hydro-7H-pyrazolo[4,3-

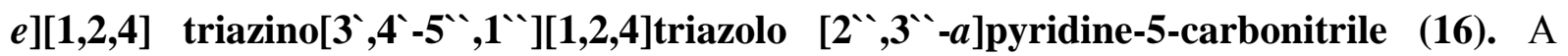
mixture of 4 (1.70 g, $0.005 \mathrm{~mol})$ and isatine $(0.74 \mathrm{~g}, 0.005 \mathrm{~mol})$ in ethanolic $\mathrm{NaOH}(5 \%, 100$ $\mathrm{mL}$ ) was refluxed for 4 hours, the reaction mixture was cooled and acidified with diluted $\mathrm{AcOH}$. The solid so formed was filtered and crystallized from DMF to give $\mathbf{1 6}$ as pale brown crystals, yield

$55 \mathrm{~g}(66 \%), \mathrm{mp}>300^{\circ} \mathrm{C}$. UV $\lambda_{\max }(\log \varepsilon): 435(2.6), 352$ (4.15), $275 \mathrm{~nm}(4.2) .\left(\mathrm{KBr}, \mathrm{cm}^{-1}\right)$ : 3467, 3309, $3207\left(2 \mathrm{NH}_{2}, \mathrm{NH}\right), 2210(\mathrm{C} \equiv \mathrm{N}), 1726(\mathrm{C}=\mathrm{O}), 1628(\mathrm{C}=\mathrm{N}) .{ }^{1} \mathrm{H}$ NMR $(\delta$, DMSO$\left.d_{6}\right): 3.47\left(\mathrm{bs}, 2 \mathrm{H}, \mathrm{NH}_{2}\right), 5.40(\mathrm{~s}, 2 \mathrm{H}, 2 \mathrm{NH}), 7.27-7.94(\mathrm{~m}, 8 \mathrm{H}, \mathrm{Ar}-\mathrm{H}), 12.06 \mathrm{ppm}(\mathrm{s}, 1 \mathrm{H}$, $\mathrm{NH} \rightleftharpoons \mathrm{OH}$ of 1,2,4-triazinone). MS (Int.\%): 469 (0.99), 322 (1.84), 285 (100), 270 (10.68), 211 (4.24), 146 (12.51), 113 (52.33), 93 (16.40), 67 (28.14). Anal. Calcd. for $\mathrm{C}_{22} \mathrm{H}_{13} \mathrm{ClN}_{10} \mathrm{O}$ (468.87): C, 56.36; H, 2.80; N, 29.86. Found C, 56.11; H,3.66; N, 29.84

Acetylation of 15- formation of 17. A mixture of $15(0.45 \mathrm{~g}, 0.001 \mathrm{~mol})$ and acetic anydride $(10 \mathrm{~mL})$ was refluxed for 2 hours. The solid obtained while hot was filtered, washed with ether and crystallized from $\mathrm{AcOH}$ to give 17 as yellow crystals, yield $0.3 \mathrm{~g}(57 \%), \mathrm{mp}>300$ ${ }^{\circ} \mathrm{C}$. IR $\left(\mathrm{KBr}, \mathrm{cm}^{-1}\right): 3422(\mathrm{NH}), 2922,2852\left(\mathrm{CH}_{3}\right), 2217(\mathrm{C} \equiv \mathrm{N}), 1720,1655(2 \mathrm{C}=\mathrm{O}), 1620$ $(\mathrm{C}=\mathrm{N})$. Anal. Calcd. for $\mathrm{C}_{26} \mathrm{H}_{15} \mathrm{ClN}_{10} \mathrm{O}_{2}$ (534.93): C, 58.38; H, 2.80; N, 26.18. Found $\mathrm{C}$, $57.81 ; \mathrm{H}, 2.56 ; \mathrm{N}, 25.85$.

Formation of $\mathbf{N}$-acetyl derivative 18. A mixture of $\mathbf{1 6}(0.94 \mathrm{~g}, 0.002 \mathrm{~mol})$ and glacial acetic acid $(40 \mathrm{~mL})$ was refluxed for 4 hours, the reaction mixture was cooled and poured onto ice. The solid obtained was filtered and crystallized from $\mathrm{AcOH}$ to give $\mathbf{1 8}$ as yellow crystals, yield 0.64 g (63\%), m.p. $>300{ }^{\circ} \mathrm{C}$. UV $\lambda_{\max }(\log \varepsilon): 384$ (3.4), 349 (4.6), $278 \mathrm{~nm}(4.8)$. IR $\left(\mathrm{KBr}, \quad \mathrm{cm}^{-1}\right): 3465,3407,3314,3143\left(2 \mathrm{NH}_{2}, 2 \mathrm{NH}\right), 2219(\mathrm{C} \equiv \mathrm{N}), 1764$ and $1671(2 \mathrm{C}=\mathrm{O})$, $1620(\mathrm{C}=\mathrm{N}), 1493,1394\left(\right.$ def. $\left.\mathrm{CH}_{3}\right) .{ }^{1} \mathrm{H} \mathrm{NMR}\left(\delta, \mathrm{DMSO}-d_{6}\right): 1.91\left(\mathrm{~s}, 3 \mathrm{H}, \mathrm{CH}_{3}\right), 5.32(\mathrm{bs}, 2 \mathrm{H}$, $\mathrm{NH}_{2}$ ), 7.48-7.94 (m, 8H, Ar-H), 9.63 (s, 1H, -NHCO-), 11.88 (bs, 1H, NH $\left.\rightleftharpoons \mathrm{OH}\right)$. Anal. 
Calcd. for $\mathrm{C}_{24} \mathrm{H}_{15} \mathrm{ClN}_{10} \mathrm{O}_{2}$ (510.90): C,56.37; H,2.94; N, 27.40. Found C, 55.87; H, 2.75; N, 26.98

Formation of bi-acetyl derivative 19. A mixture of 16 ( $0.94 \mathrm{~g}, 0.002 \mathrm{~mol})$ in glacial acetic acid $(50 \mathrm{~mL})$ and acetic anhydride $(1 \mathrm{~mL})$ was refluxed for 8 hours, the reaction mixture was cooled and poured onto ice. The solid obtained was filtered and crystallized from acetic acid to give 19 as yellow crystals, yield $0.61 \mathrm{~g}(58 \%), \mathrm{mp}>30{ }^{\circ} \mathrm{C}$. IR $\left(\mathrm{KBr}, \mathrm{cm}^{-1}\right): 3502,3397$, 33192, (3NH), $2924\left(\mathrm{CH}_{3}\right), 2234(\mathrm{C} \equiv \mathrm{N}), 1740,1702,1658(3 \mathrm{C}=\mathrm{O}), 1596(\mathrm{C}=\mathrm{N}), 1495,1455$ (def.

$\left.\mathrm{CH}_{3}\right)$.

${ }^{1} \mathrm{H}$ NMR ( $\delta$, DMSO-d $\left.d_{6}\right): 1.56\left(\mathrm{~s}, 3 \mathrm{H}, \mathrm{CH}_{3}\right), 1.92\left(\mathrm{~s}, 3 \mathrm{H}, \mathrm{CH}_{3}\right), 7.35-7.64(\mathrm{~m}, 8 \mathrm{H}, \mathrm{Ar}-\mathrm{H}), 9.65$ (bs, $1 \mathrm{H}, \mathrm{NH}$ ) and 13.09 (bs, $2 \mathrm{H}, 2 \mathrm{NH}$ ). Anal. Calcd. for $\mathrm{C}_{26} \mathrm{H}_{17} \mathrm{ClN}_{10} \mathrm{O}_{3}$ (522.94): C, 59.66; $\mathrm{H}$, 3.25; N, 26.77. Found C, 59.42; H, 3.36; N, 26.74.

\section{References}

1. Elnagdi M. H.; Ghozlan S. A.; Abdel-Razik F. M.; Maghraby A. S. Chem. Synop 1991, 5, 116.

2. Attaby F. A.; Eldin S. M.; Abdel-Razik F. M. Phosphorus, Sulfur Silicon Relat.Elem. 1995, 21, 106.

3. Asadov Kh. A.; Burangulova R. N.; Guseninov F. H.; Gilmanov R.Z.; Phaljachov I. Ph. Chem. Heterocycl.Compounds 2003, 39, 392.

4. Miletin M.; Hartl J.; Dolezal M.; Odlerova Z.; Kralova K.; Machacek M. Molecules 2000, 5, 208.

5. Abdel-Rahman A.; Bakhite E. A.; Al-Laifi E. A. J. Chin. Chem. Soc. 2002, 49, 223.

6. Rao C. S.; Venkaleswarlu V.; Achaiah G. Bioorg Med. Chem. Lett. 2006, 16, 2134.

7. Todd A.H. Brit. 1 1970, 203, 149; Chem. Abstr. 1970, 73, 120508 b.

8. Younghale G.A. U.S. 1980, 288, 440; Chem. Abstr. 1982, 96, 6596c

9. Li F.; Feng Y.; Meng Q.; Li W.; Wang Q.; Tao F. ARKIVOC 2007, (i), 40.

10. Abdel-Rahman R.M., Morsy J.M.; El-Edfawy S.; Ameneand H. A. Pharmazie 1999, 54, 347.

11. Abdel-Rahman R.M.; Morsy J. M.; Hanafy F.; Abdel-Salam H.A. Pharmazie 1999, 54, 3

12. El-Gendy Z.; Abdel-Rahman R.M. Indian J. Heterocycl. Chem. 1995, 4, 295.

13. Abdel-Rahman, R. M. Trends Heterocycl. Chem. (India) 2002, 8, 187.

14. Abdel-Rahman, R. M. Phosphorus, Sulfur, Silicon Relat. Elem. 2000, 166, 315.

15. Abdel-Rahman, R. M. Trends Heterocycl. Chem.(India) 1999, 6, 126.

16. Abdel-Rahman, R. M. Pharmazie 2001, 56, 275.

17. Abdel-Rahman, R. M. Pharmazie 2001, 56, 195.

18. Abdel-Rahman, R. M. Pharmazie 2001, 56, 18.

19. Abdel-Rahman, R. M. Pharmazie 1999, 54, 791. 
20. Abdel-Rahman, R. M.; El-Gendy, Z.; Mahmoud, M. B. Indian J. Chem. 1990, $29 B$, 352.

21. Abdel-Rahman, R. M.; Islam, M. I. E.; El-Gendy, Z. J. Indian Chem. Soc. 1991, 68, 621.

22. Abdel-Rahman, R. M.; El-Gendy, Z.; Fawzy, M. M. Asian J. Chem. 1992, 4(2), 364.

23. Abdel-Rahman, R. M.; Fawzy, M. M.; El-Gendy, Z. Asian J. Chem. 1992, 4(3), 534.

24. Abdel-Rahman, R. M.; Seada, M.; El-Gendy, Z.; Islam, I. E.; Mahmoud, M. B. Farmaco 1993, 48(3), 407.

25. Abdel-Rahman, R.M.; Abdel-Halim, A.M.; Ibrahim, S. S.; Mohamed E. A. J. Chem. Soc. (Pakistan) 1987, 9(4), 523.

26. Shawali, A. S.; Gomha, S. M. Tetrahedron 2000, 58, 8559.

27. Ismail, M. M. Chem. Pap. 2001, 55(4), 241.

28. Eladawy, M. A. Sulfur Lett. 1990, 11, 1, Chem. Abstr. 1990, 118, 115260d.

29. Zaher, H. A.; Abdel-Rahman, R. M.; Abdel-Halim, A. M. Indian J. Chem. 1987, 26B, 110.

30. Fathy, N. M.; Aly, A. S.; Hassan, N. A.; Abu-Zied, K. M.; Abdel-Fatah, M. Egypt J. Chem. 1997, 40(2), 117.

31. Abdel-Halim, A. M.; El-Gendy, Z.; Abdel-Rahman, R. M. Pharmazie 1995, 50, 726.

32. Sztanke, K., Fidecka, S.; Kedzierska, E.; Karczmarzyk, Z.; Pihlaja K.; Malosuk, Eur. J. Med. Chem. 2005, 40, 127.

33. Gould, J. C., Bowie, J. M. Edinb. Med. J. 1952, 59, 198.

34. Singh, A.; Latita, R.; Dhakarey, R.; Saxena, G. J. Indian. Chem. Soc. 1996, 73, 339.

35. Al-Najjar, A. A. A.; Amer, S. A.; Riad, M.; Elghamy, I.; Elnagdi, M. H. J. Chem. Res.(S) 1996, 296. 